\title{
NOVA METODOLOGIA PARA O CÁlCULO DE REDES DE TUBULAÇÃO COM ANÉIS
}

\author{
R. RAONI L.B ${ }^{1}$, A. R. SECCHI ${ }^{1}$ e E. C. BISCAIA ${ }^{1}$ \\ ${ }^{1}$ Universidade Federal do Rio de Janeiro, PEQ-COPPE \\ E-mail: rafael_raoni@hotmail.com.br
}

\begin{abstract}
RESUMO - O presente trabalho apresenta um novo método para determinar as vazões em redes de tubulação com anéis, que consiste na formulação de um sistema não linear de equações algébricas. Como variáveis de busca são utilizadas vazões e pressões das tubulações de entrada da rede e como equações do sistema é utilizada a diferença de pressão das tubulações que se encontram em um nó e a diferença entre a pressão calculada e especificada dos nós de saída da rede. Na modelagem do problema, o balanço de massa nos nós ( $1^{\mathrm{a}}$ Lei de Kirchoff) deve ser atendido e, após sua resolução, o somatório da perda de carga em qualquer circuito fechado deve ser nulo ( $2^{\mathrm{a}}$ Lei de Kirchoff). A metodologia tem como vantagem a não necessidade de identificação e escolha dos circuitos da rede para a resolução do problema. Os resultados obtidos foram satisfatórios para redes de grande e pequena dimensão e em circuitos fechados de sistemas de recirculação de bombas, frequentemente encontrados em terminais de transferência de petróleo. Sua utilização se torna de grande importância para o cálculo de instalações industriais de tubulações que formam anéis, já que a metodologia desenvolvida pode ser aplicada na busca de qualquer variável do problema.
\end{abstract}

\section{INTRODUÇÃO}

O objetivo em um problema de rede é a obtenção da vazão e pressão em todas as suas tubulações e nós. Para sua resolução, as características físicas das tubulações e especificação de algumas variáveis são necessárias. Muitos problemas de rede são facilmente resolvidos através de um procedimento modular sequencial, entretanto, quando há diferentes tubulações escoando para o mesmo nó ou circuitos fechados na rede, alguns outros métodos devem ser utilizados.

Para a resolução de um problema de rede com anéis, é comum o uso das duas leis de circuitos de Kirchoff, desenvolvida para resolução de problemas elétricos. A primeira e a segunda lei são equivalentes à equação da continuidade e de conservação de energia respectivamente (Martinez and Puigjaner, 1988), e podem ser descritas da seguinte maneira:

$1^{\text {a }}$ : A soma algébrica das correntes em um nó da rede de condutos é zero.

$2^{\mathrm{a}}$ : A soma direta do potencial elétrico (voltagem) ao redor de qualquer circuito fechado é zero. 


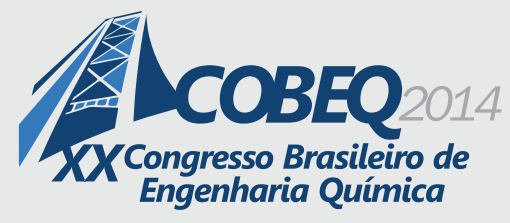

19 a 22 de outubro de 2014
Florianópolis/SC

Cross (1936) propôs um dos mais relevantes métodos de resolução, baseado na linearização das equações não lineares obtidas pela aplicação das leis de Kirchoff. Gay e Middleton, 1970, relataram que a metodologia é essencialmente um método de relaxação adaptado para resolução manual. O método se baseia no atendimento inicial a uma das Leis de Kirchoff, para a obtenção de um sistema não linear de equações, resultando em duas metodologias: Método de Balanço de Vazão e Método de Balanço de Pressão, que atendem a primeira Lei e a segunda Lei.

O Método de Balanço de Pressão é apropriado para resolver problemas com especificações de vazões nas entradas e saídas da rede, porém, a partir do desenvolvimento das equações de pseudoloop (Streeter e Wyle, 1982), é possível resolver também problemas com especificações de pressões. O método tem como principal desvantagem a necessidade de identificação e seleção dos circuitos representativos da rede (Gay e Middletont, 1970; Rao, 1987), já que o número de circuitos possíveis de serem escolhidos é normalmente maior que o número de circuitos necessários para a resolução do problema. Atualmente esta escolha pode ser realizada via grafos, sendo de conhecimento comum que os circuitos representativos devem ser classificados como circuitos independentes (Jha, 2007).

O Método de Balanço de Vazão é apropriado para resolver problemas com especificação de pressão nas entradas e saídas, sendo, a princípio, necessária uma equação de perda de carga com vazão explicita. Alguns autores resolvem o problema com a correção iterativa da pressão nos nós, a partir da resolução de um sistema não linear de equação (Rao, 1987), outros resolvem via técnica de otimização heurística Simulated Annealing (Yeh e Lin, 2011).

Outros métodos para a resolução do problema, chamados de métodos híbridos, foram desenvolvidos. Em tais métodos não há necessidade do atendimento inicial de uma das Leis de Kirchoff, como mostrado por Osiadacz (1987). Nessa metodologia, ambos os grupos de equação, balanço de massa e equações de circuito, são convergidos simultaneamente, o que resulta em um problema com mais equações e variáveis de busca.

Apesar da maior atenção, na literatura, ao problema de rede de distribuição de água, a metodologia de resolução é importante para qualquer tipo de problema com arranjo de tubulações com anéis e escoamento de qualquer produto. Tais arranjos são encontrados em plantas industriais, no by-pass de equipamentos, ou em redes de distribuição de gás em cidades. Neste cenário, atualmente, é muito comum encontrar diferentes metodologias, cada uma com sua particularidade e melhor aplicabilidade para resolução dos problemas de redes com anéis (Brkic, 2011). No presente trabalho é apresentado um novo método para determinar as vazões em redes de tubulação com anéis, que consiste na formulação de um sistema não linear de equações algébricas.

\section{METODOLOGIA}

Comparando o Método de Balanço de Pressão de Hardy-Cross com outros já apresentados, este é o mais simples de ser implementado em função da forma normal de apresentação das equações de perda de carga, com perda de carga explícita, e da intuitiva utilização do balanço de massa em qualquer modelo da rede. Com o uso deste método não há erro no balanço de massa da rede. A metodologia aqui descrita formula o problema com modelos, para tubulações e nós, que atendem o 
balanço de massa. Após esta modelagem, é possível a conexão entre estes, o que resulta na formulação da rede, intitulada Flowsheet.

Em uma rede de tubulações sem anéis, com X entradas e Y saídas, são necessárias especificar X + Y variáveis independentes para obter um problema sem graus de liberdade. É possível verificar que uma rede com anéis possui menos entradas ou saídas que outra rede sem anéis com o mesmo número de tubulações. Assim, em uma rede de tubulações com anéis, quando o número de variáveis independentes especificada é igual ao número de entradas e saídas da rede, não há outra variável independente a ser especificada. Nesta situação, é obtido um problema com menos graus de liberdade.

A equação encontrada em Martinez (1998), Equação (1), pode ser utilizada para identificar o número de graus de liberdade de uma rede de tubulações. Em que $T$ é o número de tubulações, $M$ o número de graus de liberdade e $N$ o número de nós da rede. Atualmente, as equações de circuitos são utilizadas para eliminar estes graus de liberdade, entretanto, a diferença entre a pressão final de tubulações que se encontram em um mesmo nó pode ser utilizada no lugar destas equações.

$$
T=M+N-1
$$

\subsection{Exemplo Introdutório}

Para introduzir a metodologia, uma rede de tubulação com anel, encontrada em Streeter e Wylie (1982), apresentada na Figura 1, é usada.

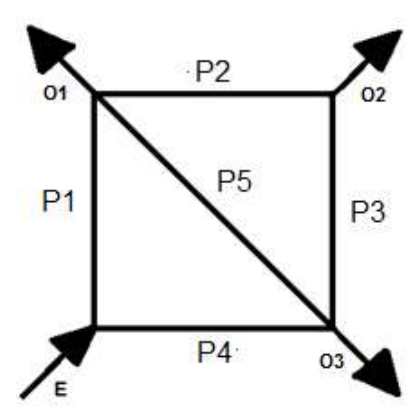

Figura 1 - Rede de tubulação com anel simples.

A partir da Equação (1), é verificado que o número de graus de liberdade do problema é dois. Para formular as equações de circuito ou de diferença de pressão, é necessária, inicialmente, a estimativa do sentido dos fluxos nas tubulações, assim como apresentado na Tabela 1. A partir destes sentidos, é possível a formulação de duas equações de diferença de pressão, Equações (2) e (3), onde $P f_{i}$ é a pressão no final da tubulação $i$. Tais sentidos devem ser interpretados como a forma em que os modelos foram conectados para a formação do Flowsheet.

Verifica-se que, o número de equações de diferença de pressão formadas é igual ao número de graus de liberdade do problema, enquanto é possível a formulação de três equações de circuitos. 
Tabela 1 - Estimativa das direções dos fluxos nas tubulações.

\begin{tabular}{c|c|c|c|c}
\hline P1 & P2 & P3 & P4 & P5 \\
\hline$\uparrow$ & $\rightarrow$ & $\uparrow$ & $\rightarrow$ & $\downarrow$ \\
\hline
\end{tabular}

$P f_{4}-P f_{5}=0$

$P f_{2}-P f_{3}=0$

\subsection{Análise do procedimento de resolução}

Assim como mostrado por Boston et al. (1993), no campo de simuladores de processo, o tipo de modelagem pode ser classificado grosseiramente em dois grupos: modular sequencial e orientado a equação. $\mathrm{O}$ procedimento sequencial é aquele em que todo problema pode ser resolvido através das especificações das variáveis de entrada do primeiro equipamento, com uma resolução sequencial dos modelos dos equipamentos adjacentes. A simulação orientada a equações agrupa todas as equações, presentes internamente nos modelos e em suas conexões, para resolvê-las simultaneamente. Assim, através desse procedimento, há a possibilidade de avaliar quaisquer variáveis do problema.

$\mathrm{Na}$ metodologia proposta, o problema deve ser resolvido com um procedimento sequencial para que, a partir deste, haja a formação do sistema não linear de equações representativo da rede. Quando a resolução sequencial chega a um nó com $X$ saídas $(X>1)$ é necessário estimar $X-1$ variáveis de vazão, sendo a $X$ 'ésima variável de vazão obtida através do balanço de massa. Quando diferentes tubulações terminam em um único nó, $Y$ tubulações, por exemplo, deverão ser formadas $Y-1$ equação de igualdade de pressão. A resolução de tais equações e busca de tais variáveis, obtidas a partir do procedimento sequencial, formam o sistema não linear de equações e precisam ser convergidas simultaneamente.

Conforme dito anteriormente, a partir do procedimento sequencial, é necessária a especificação das variáveis de entrada do primeiro equipamento a fim de serem obtidas as variáveis de saída do problema. Assim, nessa metodologia proposta, quando uma entrada não é especificada, esta deve ser arbitrada, e quando uma variável de saída é especificada, esta deve ser convergida. Utilizando um exemplo em que a pressão em $\mathrm{O} 1$ da rede apresentada na Figura 1 é especificada, outra equação de diferença de pressão pode ser formulada, conforme apresentado na Equação (4).

$$
P f_{1}-P O_{1}=0
$$

Esta equação substitui a equação de pseudoloop já comentada. Caso qualquer outra variável de saída seja especificada, o mesmo procedimento pode ser utilizado para sua convergência. Este procedimento de resolução pode ser classificado como modular-simultâneo, que apresenta a vantagem de convergência de qualquer variável do problema e de obter um sistema reduzido de equações não lineares, quando comparado com o procedimento orientado a equações. 


\section{9 a 22 de outubro de 2014 \\ Florianópolis/SC}

É possível verificar que as Equações (2) e (3) são equações de circuito, e a Equação (4) é uma equação de pseudoloop. Esta análise mostra que, com o uso das equações de diferença, o problema será resolvido a partir das mesmas equações utilizadas nas demais metodologias existentes.

O sistema formulado pode ser convergido pelo método de Newton-Raphson ou ser adaptado para um problema de otimização, a ser resolvido por métodos heurísticos, com as mesmas variáveis de busca e função objetivo igual ao somatório do quadrado das equações de diferença de pressão. Tal adaptação para um problema de otimização pode ser utilizada a fim de serem geradas melhores estimativas iniciais para a variável de busca para a resolução do sistema não linear de equações, já que é sabido que os métodos de otimização heurísticos apresentam alto custo computacional e os sistemas de equações não lineares podem apresentar dificuldade de convergência em função de suas estimativas iniciais.

Sendo o objetivo do problema a obtenção da vazão, que também significa a obtenção do sentido do fluido nas tubulações, há a possibilidade de esta escolha inicial do sentido ser contrária à da solução do problema. Com isso, os modelos utilizados devem ter a capacidade de lidar com valores de vazão negativa, o que, nas tubulações, deve gerar quedas de pressão negativa. A obtenção de uma vazão negativa não é um problema para o desenvolvimento da metodologia.

\section{ESTUDO DE CASO E DISCUSSÕES}

A fim de apresentar a metodologia proposta, é resolvido um problema comumente encontrado em instalações industriais, com bombas de grande capacidade escoando petróleo, que visa exemplificar a aplicabilidade da metodologia na resolução de uma rede. $\mathrm{O}$ arranjo apresentado na Figura 2 é formado pelo sistema de recirculação de dois grupos de bombas, conectadas em série, com sucção em um tanque de petróleo.

Este arranjo é encontrado em instalações com bombas de grande capacidade (grande vazão e alta pressão de descarga), que necessitam de bombas boosters em sua sucção, em função de seu alto NPSH requerido. A operação de recirculação é necessária na partida da bomba, a fim de minimizar sua potência requerida e evitar problemas elétricos.

A fim de adequar a pressão de descarga com a pressão de suç̧ão da bomba, é necessária a previsão de um orifício de restrição para ser obtida a devida queda de pressão no sistema. A equação representativa deste acidente pode ser encontrada na ISO-5167-2 e, no problema estudado, foi utilizado dois orifícios de restrição alinhados em série. Para o cálculo do comprimento equivalente das linhas, foi utilizada uma equação empírica que faz uso de um fator de complexidade, conforme apresentado na técnica de projeto PRJ-TEC-N ${ }^{\circ}-3083-30-P 0-I T-002 / R 1$.

Analisando a rede, é possível identificar duas tubulações que terminam em um mesmo nó, as tubulações 1 e 13, e outra que tem sua pressão final definida, a tubulação 10, que na solução do problema deve ser igual à pressão da coluna do tanque. Na Tabela 2 são apresentadas as equações e as variáveis de busca do problema e na Tabela 3 suas especificações. 


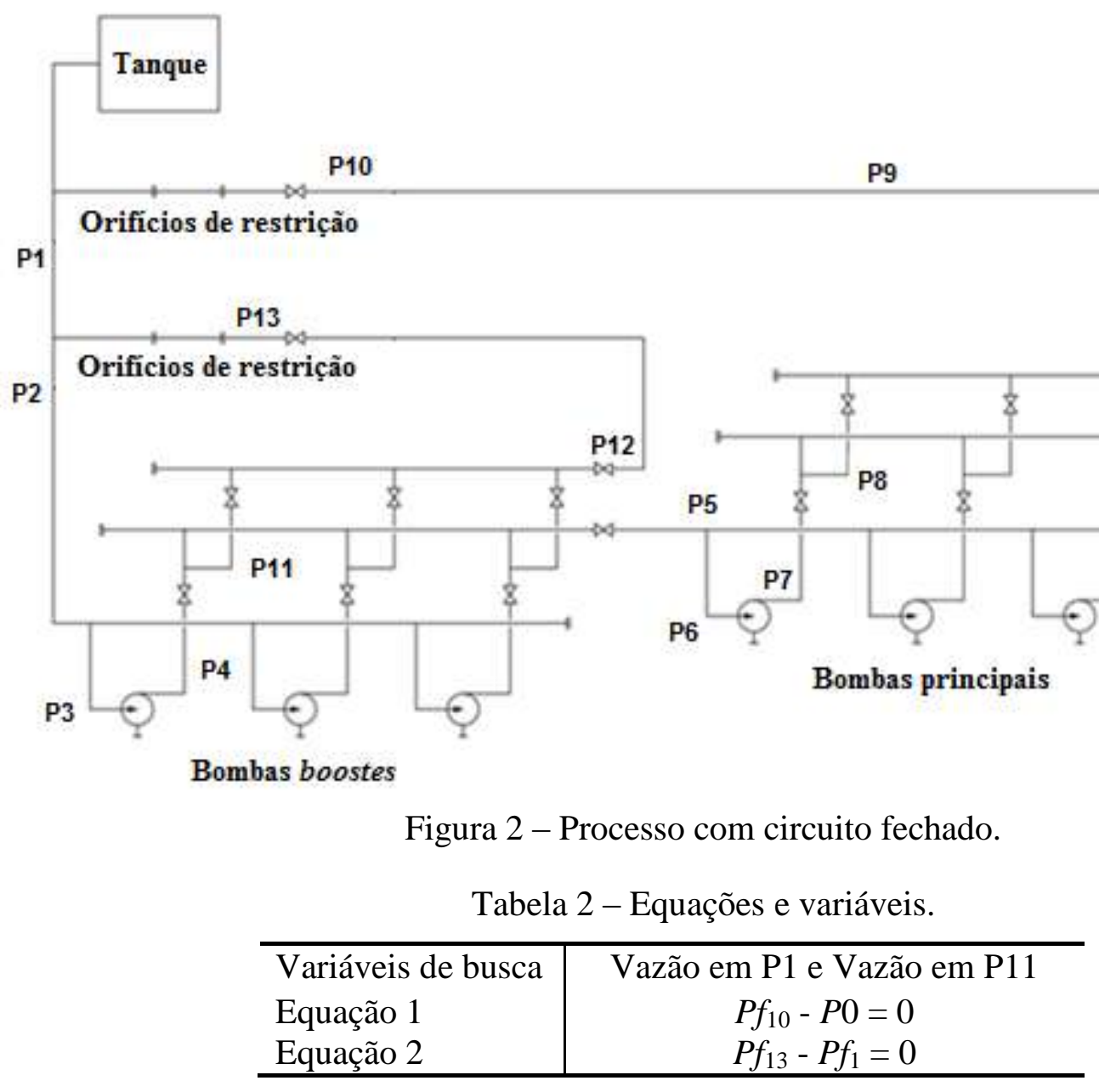

Tabela 3 - Especificações da simulação.

\begin{tabular}{|c|c|}
\hline P0 (Coluna de petróleo) & $12 \mathrm{~m}$ \\
\hline Equação de perda de carga & Darcy-Weisbach \\
\hline Rugosidade & $0,04572 \mathrm{~mm}$ \\
\hline Comprimento reto (para as 11 tubulações) & {$\left[\begin{array}{lllllllllllll}20 & 30 & 10 & 10 & 70 & 16,5 & 6 & 15,9 & 80 & 10 & 10 & 20 & 10\end{array}\right] \mathrm{m}$} \\
\hline Diâmetro (para as 11 tubulações) & {$\left[\begin{array}{lllllllllllll}55 & 55 & 29 & 23 & 41 & 23 & 19 & 23 & 9 & 7 & 15 & 7\end{array}\right]$ in } \\
\hline Fator de complexidade & 1 \\
\hline Viscosidade & $206,14 \mathrm{cP}$ \\
\hline Equação da bomba principal $\left(\mathrm{Q}[=] \mathrm{m}^{3} / \mathrm{h}\right)$ & $-4 e-12 Q^{4}-2 e-8 Q^{3}+2 e-05 Q^{2}-0,0302 Q+919,99 m$ \\
\hline Vazão mínima da bomba principal & $720 \mathrm{~m}^{3} / \mathrm{h}$ \\
\hline Equação da bomba booster $\left(\mathrm{Q}[=] \mathrm{m}^{3} / \mathrm{h}\right)$ & $-9 e-13 Q^{4}+3 e-9 Q^{3}-9 e-6 Q^{2}+0,0056 Q+141,77 m$ \\
\hline Vazão mínima da bomba booster & $320 \mathrm{~m}^{3} / \mathrm{h}$ \\
\hline Diâmetro dos orifícios das bombas & $0,0660 \mathrm{~m}(2,6 \mathrm{in})$ \\
\hline Diâmetro dos orifícios das bombas booster & $0,0381 \mathrm{~m}(1,5 \mathrm{in})$ \\
\hline
\end{tabular}


O problema foi formulado no software Matlab, sendo utilizada a função fsolve para a resolução do sistema de equações não lineares formado. Foi utilizado o valor de $1 \mathrm{~kg} / \mathrm{h}$ como estimativa inicial para as variáveis de busca e um critério de convergência de $10^{-8}$. O resultado da simulação é apresentado na Tabela 4 e, a fim de possibilitar uma melhor análise dos resultados, o valor da função objetivo do problema, calculada a partir do resultado obtido, também é apresentado.

Tabela 4 - Resultados do problema - Processo com circuito fechado.

\begin{tabular}{c|c|c|c}
\hline Variáveis & Valor & Variáveis & Valor \\
\hline$P f_{1}$ & $1,1240 \mathrm{kgf} / \mathrm{cm}^{2}$ & $Q_{1}$ & $754,8448 \mathrm{~m}^{3} / \mathrm{h}$ \\
$P f_{2}$ & $1,1234 \mathrm{kgf} / \mathrm{cm}^{2}$ & $Q_{2}$ & $852,5290 \mathrm{~m}^{3} / \mathrm{h}$ \\
$P f_{3}$ & $1,1213 \mathrm{kgf} / \mathrm{cm}^{2}$ & $Q_{3}$ & $852,5290 \mathrm{~m}^{3} / \mathrm{h}$ \\
$P f_{4}$ & $14,3634 \mathrm{kgf} / \mathrm{cm}^{2}$ & $Q_{4}$ & $852,5290 \mathrm{~m}^{3} / \mathrm{h}$ \\
$P f_{5}$ & $14,3597 \mathrm{kgf} / \mathrm{cm}^{2}$ & $Q_{5}$ & $754,8448 \mathrm{~m}^{3} / \mathrm{h}$ \\
$P f_{6}$ & $14,3524 \mathrm{kgf} / \mathrm{cm}^{2}$ & $Q_{6}$ & $754,8448 \mathrm{~m}^{3} / \mathrm{h}$ \\
$P f_{7}$ & $98,5520 \mathrm{kgf} / \mathrm{cm}^{2}$ & $Q_{7}$ & $754,8448 \mathrm{~m}^{3} / \mathrm{h}$ \\
$P f_{8}$ & $97,8696 \mathrm{kgf} / \mathrm{cm}^{2}$ & $Q_{8}$ & $754,8448 \mathrm{~m}^{3} / \mathrm{h}$ \\
$P f_{9}$ & $97,8338 \mathrm{kgf} / \mathrm{cm}^{2}$ & $Q_{9}$ & $754,8448 \mathrm{~m}^{3} / \mathrm{h}$ \\
$P f_{10}$ & $1,1244 \mathrm{kgf} / \mathrm{cm}^{2}$ & $Q_{10}$ & $754,8448 \mathrm{~m}^{3} / \mathrm{h}$ \\
$P f_{11}$ & $14,3138 \mathrm{kgf} / \mathrm{cm}^{2}$ & $Q_{11}$ & $97,6842 \mathrm{~m}^{3} / \mathrm{h}$ \\
$P f_{12}$ & $14,3081 \mathrm{kgf} / \mathrm{cm}^{2}$ & $Q_{12}$ & $97,6842 \mathrm{~m}^{3} / \mathrm{h}$ \\
$P f_{13}$ & $1,1240 \mathrm{kgf} / \mathrm{cm}^{2}$ & $Q_{13}$ & $97,6842 \mathrm{~m}^{3} / \mathrm{h}$ \\
$\Delta P$ orifício booster & $13,345 \mathrm{kgf} / \mathrm{cm}^{2}$ & $\Delta P$ bomba booster & $13,2479 \mathrm{kgf}^{2} / \mathrm{cm}^{2}$ \\
$\Delta P$ orifício principal & $96,2802 \mathrm{kgf} / \mathrm{cm}^{2}$ & $\Delta P$ bomba principal & $84,2071 \mathrm{kgf}^{2} \mathrm{~cm}^{2}$ \\
Função Objetivo & $1,6367 \mathrm{e}-012$ & $\mathrm{~N}^{\circ}$ iterações & 24 \\
\hline \multicolumn{2}{c}{}
\end{tabular}

Em um projeto de um sistema de recirculação de bombas, normalmente deseja-se calcular o diâmetro do orifício de restrição. A fim de expandir a aplicabilidade da metodologia, o mesmo problema já apresentado foi resolvido a partir da especificação de todas as variáveis de vazão, conforme resultados apresentados na Tabela 4, deixando como variáveis de busca os diâmetros dos orifícios de restrição das bombas principais e boosters. Os resultados obtidos para os valores dos diâmetros foram, conforme esperado, os mesmos valores apresentados na Tabela 3. Este resultado mostra que a metodologia apresentada é aplicável, não só para problemas de escoamento, mas para qualquer tipo de simulação de processos.

\section{CONCLUSÕES}

Em função de sua particularidade, o problema de rede com anéis não pode ser resolvido a partir de um procedimento modular sequencial. Um procedimento de resolução simultânea é necessário, a fim de serem resolvidas as equações de circuito ( $2^{\mathrm{a}}$ Lei de Kirchoff), equações que formam um sistema não linear de equações do problema.

Atualmente é comum o uso de softwares específicos para a resolução de problemas de rede, e outros para as demais simulações de processo. Com o uso das equações de diferença de pressão, que 
podem ser facilmente identificadas, deixa de ser necessária a identificação e escolha das equações de circuitos independentes, e a simulação de qualquer problema pode ser feita com o uso de um único software com capacidade de resolução simultânea. Foi verificado também, no item 3, que a equação de diferença de pressão pode ser utilizada para a busca de qualquer variável do problema.

Pode ser verificado que, a metodologia orientada a equações tem a capacidade de resolver o problema de rede com anéis a partir da introdução das equações de diferença de pressão no sistema não linear de equações. Comparando com a metodologia modular-simultânea apresentada, este procedimento tem a desvantagem de resolver todas as equações do problema simultaneamente, enquanto que a modular-simultânea resolve simultaneamente somente as equações que não podem ser resolvidas de forma sequencial, resultando em um sistema não linear de menor dimensão.

\section{REFERENCIAS}

BRKIC, D., 2011, Iterative Methods for Looped Network Pipeline Calculation, Water Resour Manage 25:2915-2987.

BOSTON, J.F.; BRITT, H.I.; TAYYABKHAN, M.T., 1993, Tackling tougher tasks, Chemical Engineering Progress 89 p.38-49.

CROSS, H., 1936, Analysis of Flow in Networks of Conduits or Conductors, University of Illinois Engineering Experiment Station.

GAY, B., MIDDLETON, P., 1970, The solution of pipe network problems, Chemical Engineering Science Vol. 26, pp. 109-123.

ISO-5167-2, Measurement of fluid flow by means of pressure differential devices inserted in circular-cross section conduits running full.

JHA, K., 2007, Automatic minimal loop extraction and initialization for water pipe network analysis, I.J. of Simulation Vol. 8 No 2.

MARTINEZ, J. M.; PUIGJANER, L., 1988, A powerful improvement on the methodology for solving large-scale pipeline networks, Computers \& Chemical Engineering, Vol. 12, No. 2/3, pp. 261-265.

OSIADACZ, A.J., 1987, Simulation and Analysis of Gas Networks, first ed., Gulf Publishing Company, Houston, TX 77252, USA.

PRJ-TEC-Nº-3083-30-P0-IT-002/R1, 2007, Engenharia de Processo, Critérios de Dimensionamento de Linhas, Projectus Consultoria Ltda.

RAO, B. V., 1987, Finite element analysis, Engineering Analysis, Volume 4 Issue 1, p. 35-39.

STREETER, V. L.; WYLIE, E. B., 1982, Mecânica dos Fluidos, Mc Graw Hill, Sétima Edição.

YEH, H. D., LIN, Y. C., 2008, Pipe Network System Analysis Using Simulated Annealing, Journal of Water Supply: Research and Technology - AQUA, 57.5. 